Giornale
di Tecniche
Nefrologiche
\& Dialitiche
Anno VII n ${ }^{\circ} 4$
Editore 1995

\title{
Glomerulonefrite membranosa: criteri istologici di evolutività
}

\author{
P.F. Palmieri, P. Santarelli, R. Summa, A. Albertazzi \\ Istituto di Clinica Nefrologica, Università di Chieti
}

egli ultimi anni sono stati effettuati numerosi studi nel tentativo di ottimizzare il trattamento farmacologico delle glomerulonefriti con risultati variabili a seconda delle casistiche (1-3). La differente risposta clinica a protocolli terapeutici molto simili impiegati nel trattamento di uno stesso tipo istologico di glomerulonefrite, fa ritenere che una diagnosi esclusivamente qualitativa possa non essere sufficiente ad un corretto inquadramento e trattamento delle nefropatie (4). Considerando la soggettività dell'osservatore è necessario non limitarsi allo studio qualitativo o semiquantitativo $(5,6)$ delle lesioni istologiche renali. L'introduzione recente nel campo della ricerca di elaboratori di immagine computerizzati (7), consistenti in sistemi in grado di elaborare l'immagine delle sezioni istologiche renali visualizzate al microscopio ottico e di riprodurle su schermo televisivo, ha stimolato lo studio di una nuova metodologia che consenta una valutazione oggettiva e quantitativa delle sezioni istologiche renali. Peraltro negli ultimi anni, sistemi computerizzati di elaborazione di immagine sono stati già impiegati con l'obiettivo di ottenere una valutazione quantitativa di depositi immunologici glomerulari evidenziati con immunoperossidasi (8).

Per quanto riguarda il trattamento della glomerulonefrite membranosa (GNM) esiste una convergenza di idee solo sulle forme di glomerulonefrite membranosa secondaria; in tali forme tutti gli Autori concordano che va trattata la malattia primitiva (neoplasie, epatite B, sifilide, tiroidite, paraproteinemia, ecc.). Per quanto riguarda le forme primitive di GNM persistono molte opinioni diverse sulle modalità di impostazione della terapia (9-15). Il nostro Gruppo ritiene (16) che il trattamento combinato di metilprednisolone e ciclofosfamide a boli sia il trattamento migliore in corso di glomerulonefrite membranosa. Con tale terapia tuttavia una discreta percentuale di pazienti presenta al follow-up a lungo termine persistenza di proteinuria e/o insufficienza renale (16).

\section{Materiali e metodi}

Abbiamo studiato retrospettivamente 81 pazienti (52 maschi, 29 femmine) affetti da glomerulonefrite membranosa accertata istologicamente. Il frustolo della biopsia renale è stato processato per immunofluorescenza, microscopia ottica e microscopia elettronica seguendo metodiche standard già pubblicate. L'osservazione dei preparati istologici ha consentito la diagnosi e l'identificazione dello stadio di evolutività secondo i criteri di Churg (19). I preparati istologici sono stati studiati anche mediante l'utilizzazione di un sistema di istomorfometria computerizzata (Fig. 1). In tutti i casi è stato eseguito uno studio clinico-laboratoristico sia all'epoca della biopsia renale che dopo un periodo di follow-up variabile da 30 a 60 mesi successivo al trattamento standard (Fig. 2). Lo studio clinico includeva la valutazione del peso corporeo, della pressione arteriosa sistodiastolica ed il riscontro di edemi. Lo studio laboratoristico comprendeva: azotemia, creatinina, protidemia totale e frazionata, IgG, IgA, IgM, C3 e C4, proteinuria die con valutazione della selettività, colesterolo totale, trigliceridi, emocromo e fibrinogeno. I criteri di esclusione dello studio sono stati: 1) presenza di glomerulonefrite membranosa secondaria, 2) terapia in atto con ACE-inibitori. Molti pazienti hanno eseguito contemporaneamente una terapia sintomatica a base di diuretici, antialdosteronici e supplementi di albumina umana endovena quando se ne sia verificata l'esigenza clinica.

\section{Protocollo terapeutico}

In tutti i casi l'approccio terapeutico (Fig. 2) è stato il seguente: metilprednisolone $1.0 \mathrm{mg} / \mathrm{kg}$ peso corporeo, per via parenterale a dì alterni per 3 mesi. L'atteggiamento terapeutico successivo è stato diverso a seconda della risposta alla terapia eseguita. In caso di remissione completa (proteinuria $<1.0 \mathrm{~g} / \mathrm{die}$ ) non 


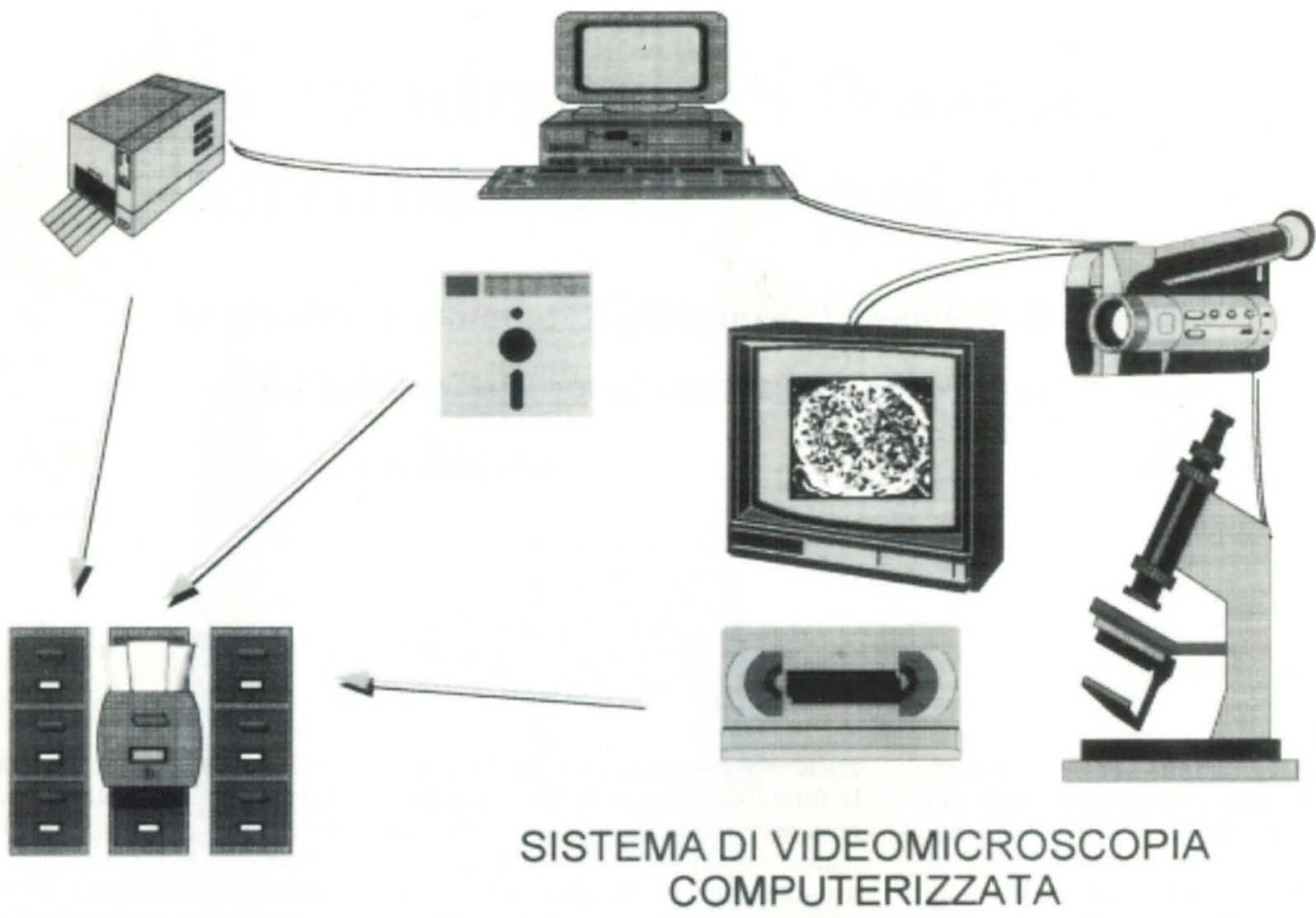

Fig. 1 - Composizione del sistema VMC: microscopio con macchina fotografica incorporata, telecamera VHS, monitor TV con videoregistratore, personal computer, stampante grafica.

èstato proseguito alcun trattamento; in caso di remissione parziale $(>1.0$ proteinuria g/die $<2.0$ ) il trattamento cortisonico sopra descritto è stato proseguito per ulteriori 3-6 mesi al termine dei quali i pazienti in remissione totale hanno sospeso ogni trattamento. In caso di mancata remissione (proteinuria $>2.0$ $\mathrm{g} /$ die) dopo il primo ciclo e in caso di parziale remissione dopo il secondo ciclo. I pazienti sono stati inseriti nel seguente protocollo terapeutico: metilprednisolone $1.0 \mathrm{~g}$ e.v. per 3 giorni consecutivi seguiti per 27 giorni da metilprednisolone $1 \mathrm{mg} / \mathrm{kg}$ peso corporeo a dì alterni; il mese successivo ciclofosfamide $1.0 \mathrm{~g}$ e.v./settimana per 4 settimane; tale schema è stato ripetuto per 3 cicli per un totale di 6 mesi. Nel corso della terapia è stata valutata l'insorgenza di effetti collaterali con particolare riguardo alla valutazione di leucopenia e/o di segni di epatotossicità. In 16 casi è stata eseguita una terapia renale di controllo.

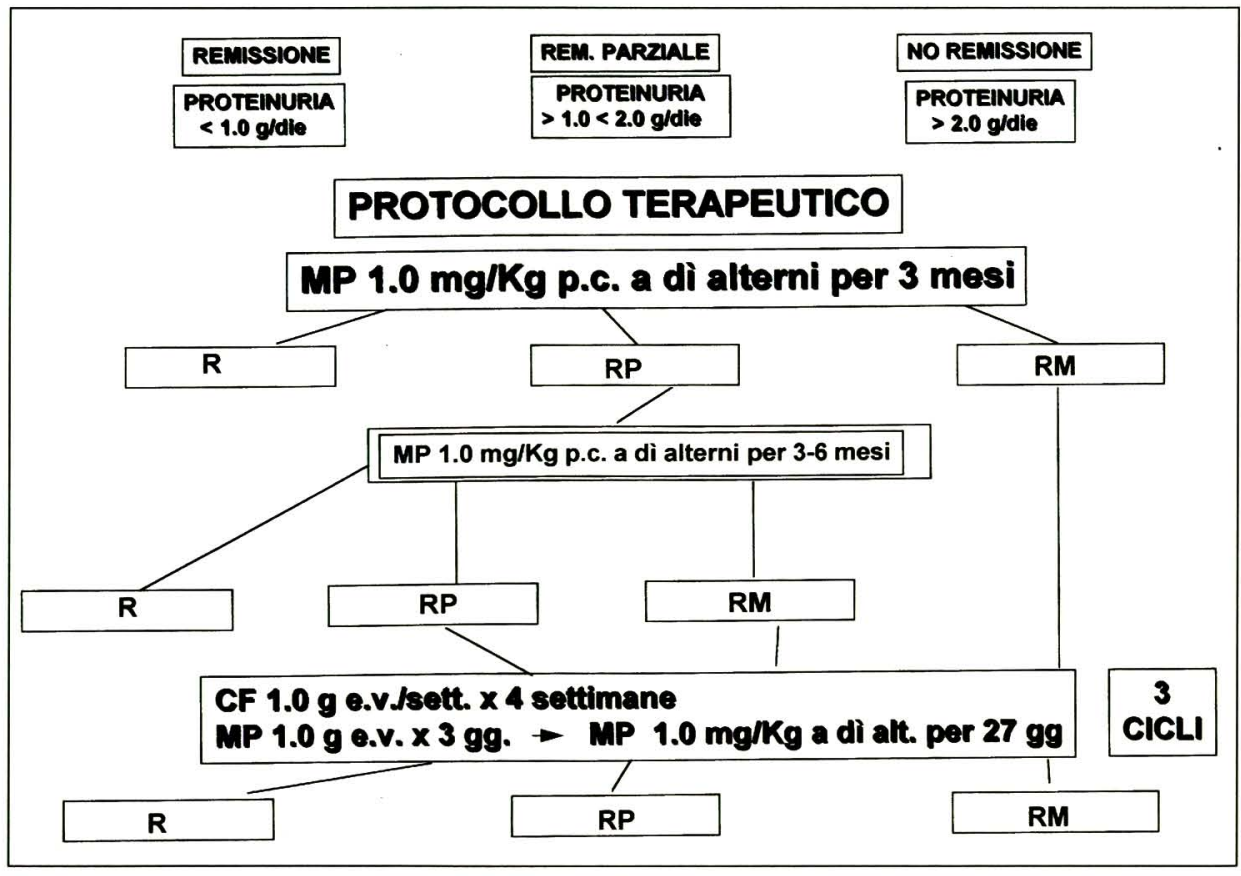

Fig. 2 - Protocollo Terapeutico impiegato nello studio. R: Remissione; RP: Remissione Parziale; RM: Mancata Remissione. 


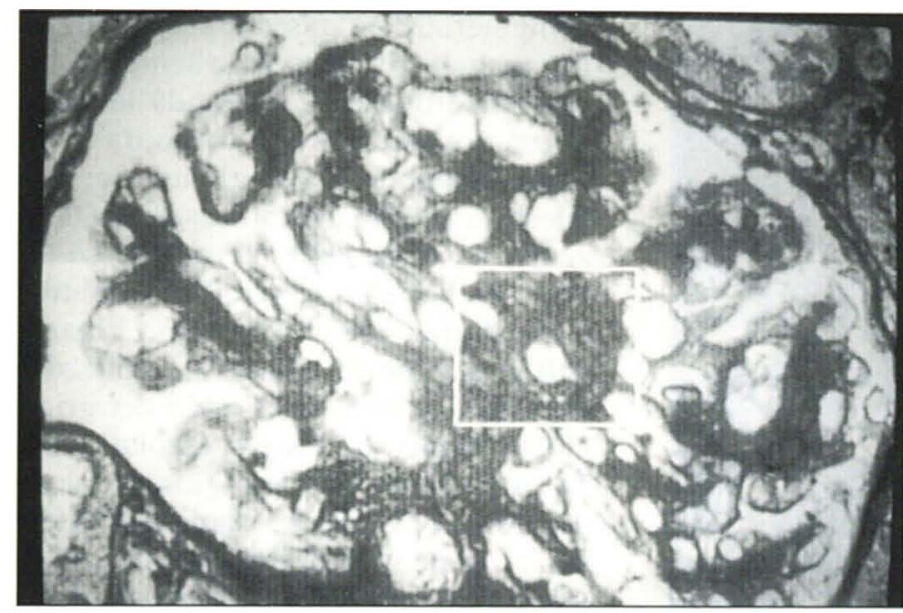

Fig. 3 - Immagine di glomerulo elaborata dal computer in 128 toni di grigio.

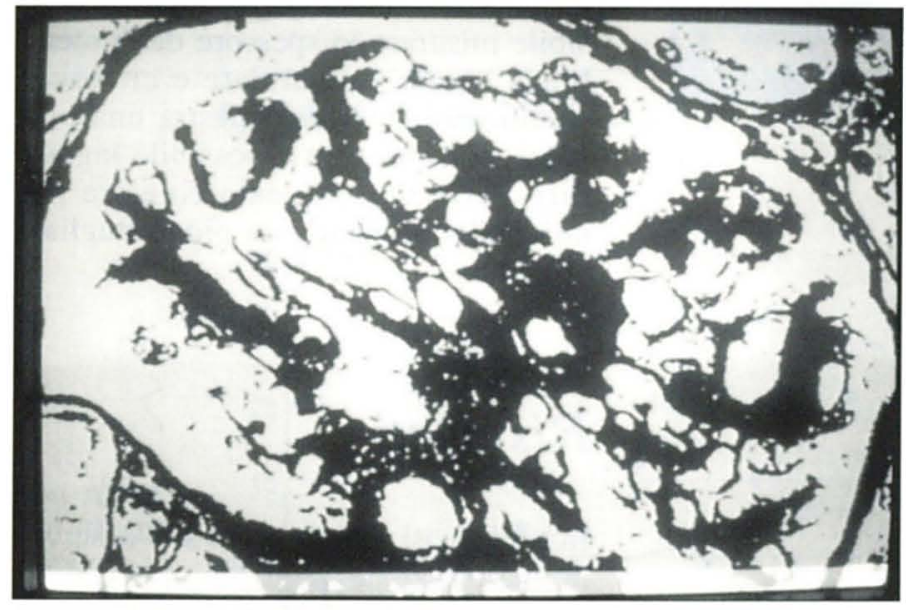

Fig. 5 - Un'elaborazione con sistema binario dell' immagine del glomerulo ottenuta impiegando solo 2 toni della scala dei grigi.

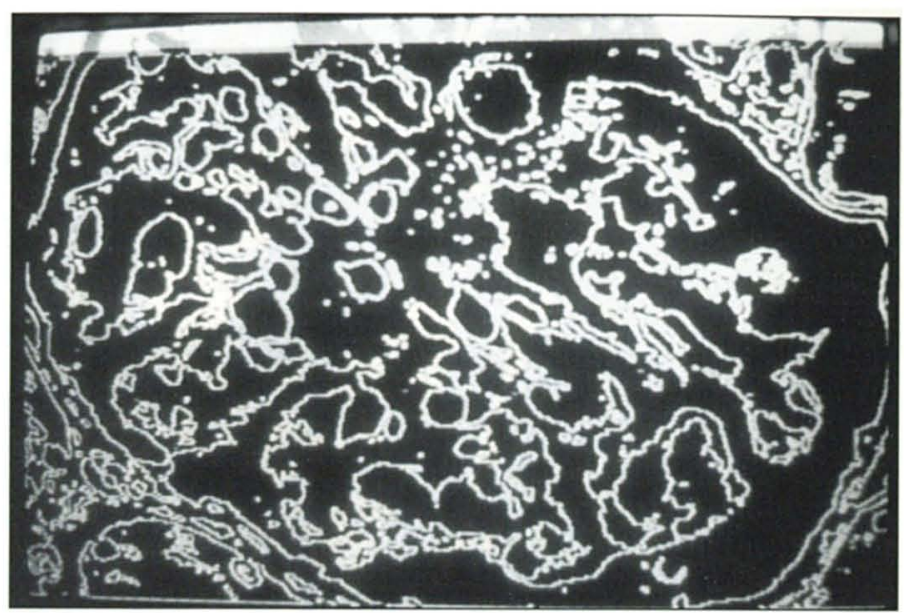

Fig. 4 - Il sistema "filtering function" consente di semplificare la struttura del glomerulo evidenziando i contorni delle membrane basali.

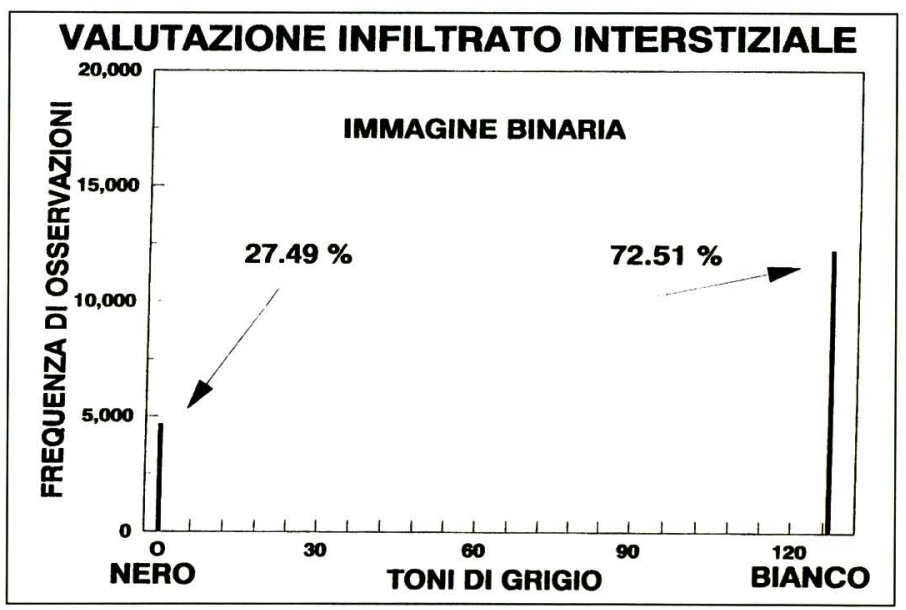

Fig. 6 - Valutazione percentuale dello spazio occupato dal nero dei nuclei cellulari in una finestra delimitante un' area interstiziale.

\section{Sistema di videomicroscopia computerizzata}

Abbiamo messo a punto un nuovo sistema di video microscopia computerizzata (VMC) che ci consente di quantizzare 1'entità delle lesioni istologiche renali. Il sistema è composto da foto-microscopio a luce polarizzata e contrasto di fase con macchina fotografica incorporata; telecamera VHS; monitor TV; videoregistratore; personal computer; stampante grafica; hardware e software Oculus 200 (Fig. 1). Le immagini microscopiche esaminate possono essere archiviate su: fotografie e diapositive, cassette video VHS, floppy e hard disk, stampe grafiche.
La possibilità di archiviazione e richiamo delle immagini consente anche 1'analisi comparativa, da parte dello stesso operatore, di preparati istologici di un singolo paziente ad un controllo bioptico successivo. Il sistema computerizzato consente di acquisire l'immagine direttamente dal microscopio tramite una telecamera e, dopo un'adeguata pre-elaborazione, di estrarre dalle immagini parametri densitometrici e morfologici. Le immagini dei glomeruli visualizzati al microscopio vengono contemporaneamente digitalizzate sul monitor televisivo ed elaborate dal software. Il sistema di acquisizione di immagine, tramite la comparsa sul monitor televisivo di un cursore mobile o di rettangoli (cioè finestre) di variabili dimensioni, consente misurazioni lineari e di superficie e fornisce la densità di luminosità in pixel di ogni punto dello schermo televisivo, con risultati che appaiono sul monitor del computer in tempo reale.

La metodica VMC ci consente di misurare: diametro flocculare, capsulare, arteriolare, tubulare; area glomerulare, capsulare, mesangiale; indice di ischemia e di sclerosi glomerulare. Consente inoltre valutazioni sulla cellularità glomerulare ed interstiziale e sullo spessore e costituzione della membrana basale. Dalle misurazioni dei diametri del flocculo glomerulare, della capsula di Bowman e delle aree di sclerosi glomerulare, siamo risaliti alle aree capsulari (A.C.), glomerulari 


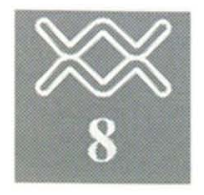

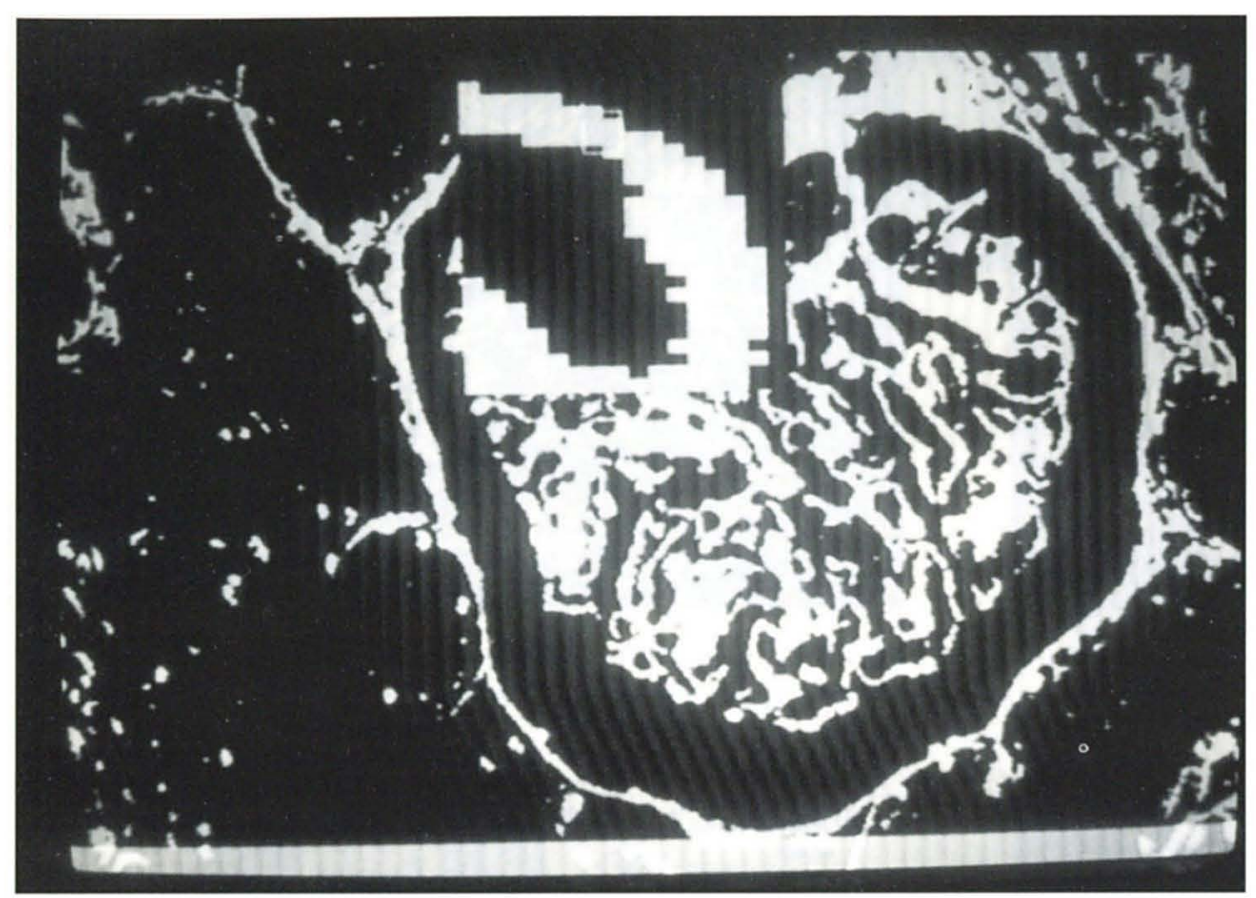

Fig. 7 - Ingrandimento di un segmento di membrana basale glomerulare.

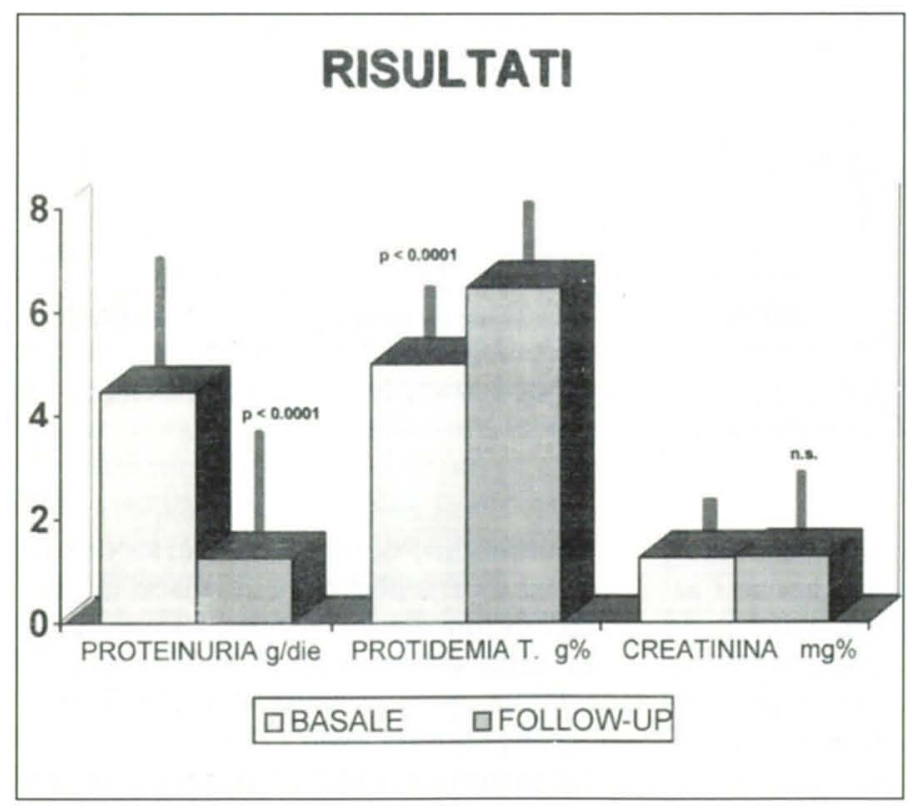

Fig. 8 - Variazioni dei principali parametri laboratoristici al termine del follow-up.

(A.G.) e di sclerosi (A.S.) impiegando la seguente formula matematica: (diametro maggiore + diametro minore $/ 4)^{2}$ $x 3.14$.

Dal rapporto A.G./A.C. abbiamo ricavato l" "indice di ischemia" (I.I.). Allo stesso modo dal rapporto tra A.S./A.C. abbiamo ricavato l'indice di sclerosi (I.S.). Oltre a semplici misurazioni l'elaboratore elettronico può fornire informazioni densitometriche sul preparato istologico renale scomponendo l'immagine proiettata sul monitor televisivo in 128 toni di grigio. Aprendo una finestra su una porzione del preparato istologico si potrà ricavare la percentuale di tessuto che presenta una stessa tonalità di grigio (Fig. 3), utilizzando colorazioni specifiche per le singole strutture istologiche. Il sistema computerizzato consente poi di filtrare l'immagine del glomerulo semplificandone la struttura ed esaltando le differenze cromatiche di contrasto tra le diverse componenti del glomerulo; con questo metodo possono essere evidenziati i contorni delle membrane basali (Fig. 4). Un'altra possibilità operativa è l'elaborazione con sistema binario dell'immagine dei glomeruli, utilizzando solo due toni della scala dei grigi, cioè il bianco ed il nero assoluti (Fig. 5). Attraverso l'elaborazione binaria possiamo delimitare un'area dell'interstizio renale sede di infiltrazione cellulare e fare una stima percentuale dello spazio occupato dai nuclei delle cellule (Fig. 6) ricavando istogrammi della cellularità interstiziale. Con la stessa tecnica si può calcolare semplicemente anche l'area mesangiale. Infine è possibile misurare lo spessore della membrana basale glomerulare e circoscrivendo mediante una finestra una porzione di membrana è possibile ingrandire varie volte questa finestra per un'analisi morfologica più dettagliata (Fig. 7).

\section{Risultati}

In tutti i casi al follow-up si è osservata una riduzione degli edemi con riduzione del peso corporeo. Non abbiamo rilevato alterazioni significative della pressione arteriosa. La proteinuria si è ridotta significativamente $(\mathrm{p}<0.001)$ e contemporaneamente si è osservato un significativo incremento $(\mathrm{p}<0.001)$ della protidemia totale e dell'albuminemia (Fig. 8). Non abbiamo osservato variazioni significative della creatininemia al termine del follow-up così come per l'emocromo e la fibrinogenemia. La colesterolemia totale e i trigliceridi si sono ridotti significativamente $(\mathrm{p}<0.001)$ al termine del trattamento. $\mathrm{Si}$ è riscontrato un incremento anche se non significativo delle IgG mentre non abbiamo registrato variazioni significative di IgA, IgM e delle frazioni del complemento. Abbiamo riscontrato una correlazione significativa ( $p<$ 0.001 ) tra i valori di creatininemia, di proteinuria/die in condizioni basali. Dei 9 pazienti con insufficienza renale in condizioni basali 4 mostravano al 


\section{FOLLOW-UP}

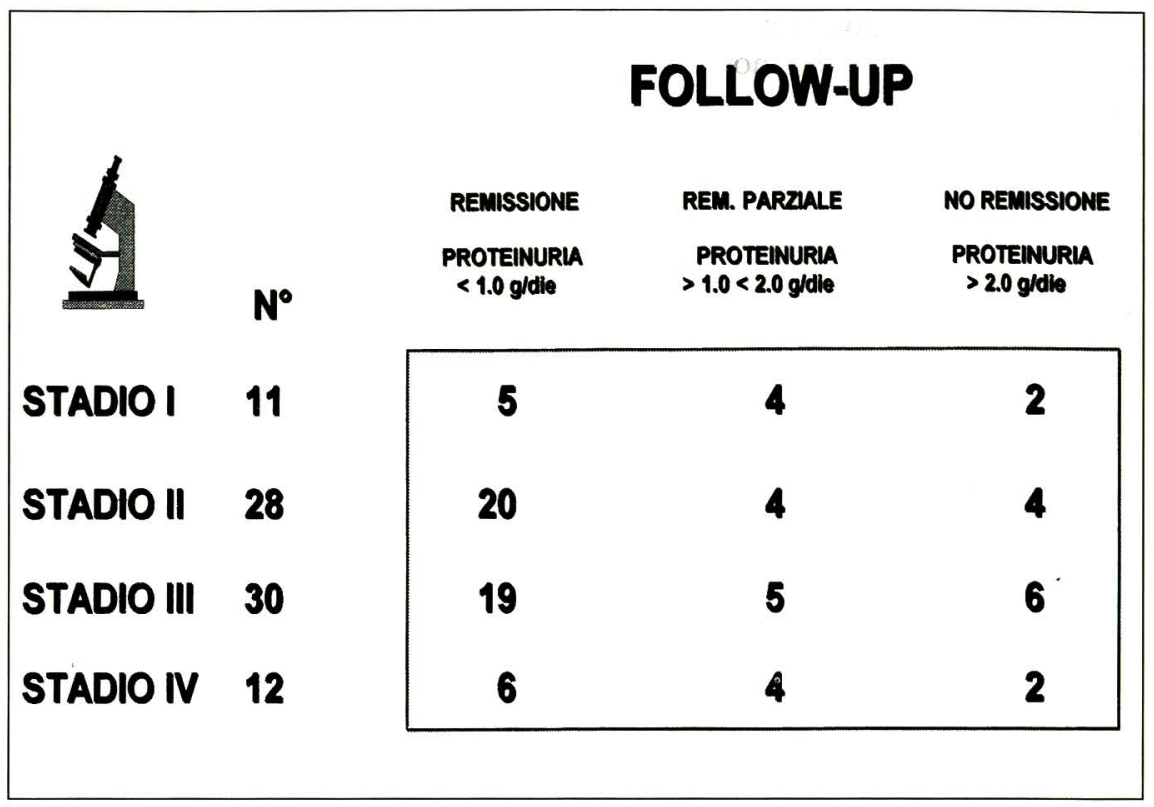

Fig. 9 - Suddivisione dei parametri in base allo stadio di GNM (secondo Churg) e andamento clinico

\section{AREA MESANGIALE vs PROTEINURIA POST}

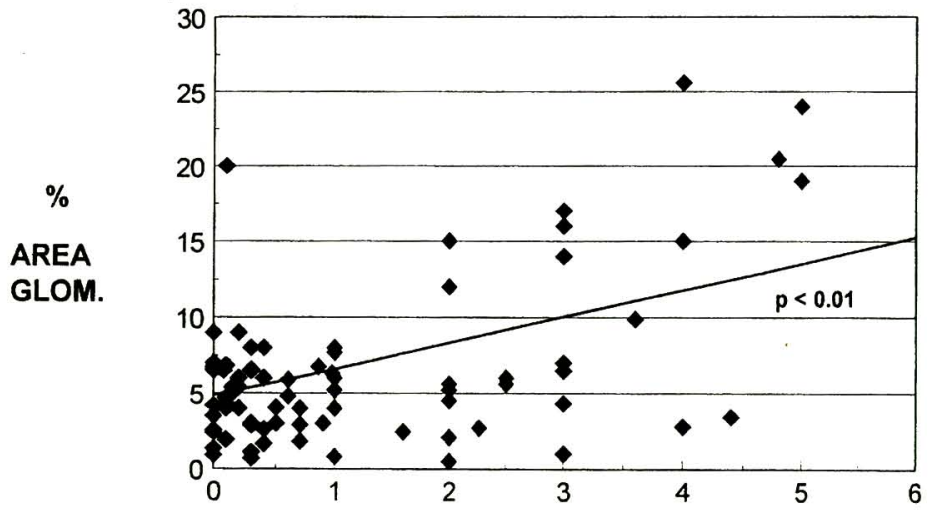

Fig. 10 - Correlazione tra area mesangiale e proteinuria post-trattamento.

$\triangle$ AREA INT. $\square$ CELL. INT.

$\%$ AREA STANDARD $\quad N^{\circ}$ cellule / AREA STANDARD

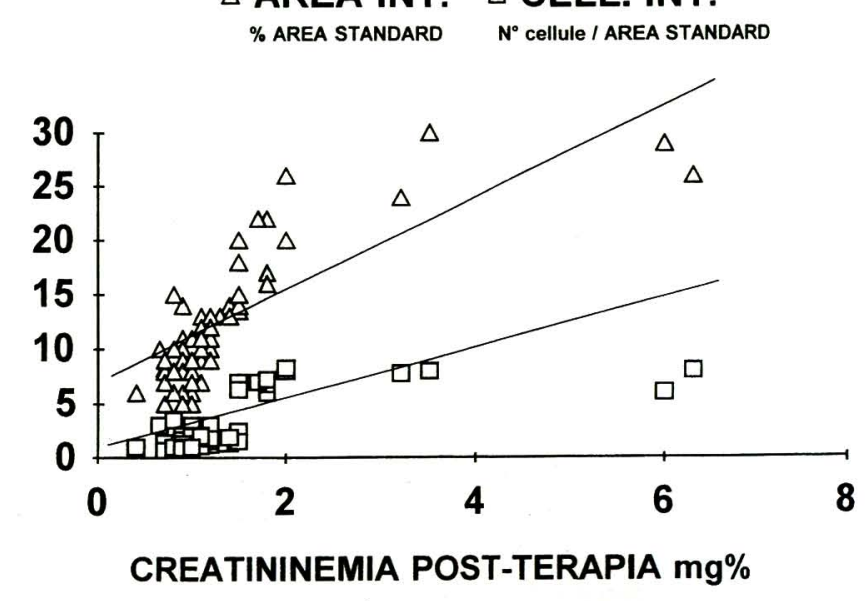


follow-up il peggioramento della funzione renale. Non abbiamo riscontrato correlazioni significative tra proteinuria pre- e post-trattamento e tra creatininemia pre- e post-trattamento. Al termine del follow-up abbiamo riscontrato una remissione completa nel $61.74 \%$ dei pazienti, una remissione parziale nel $20.98 \%$ dei pazienti, una mancata remissione del $17.28 \%$ dei pazienti. L'analisi statistica ha dimostrato assenza di correlazioni tra lo stadio di glomerulonefrite membranosa (Fig. 9) e la risposta al trattamento. Abbiamo individuato una correlazione diretta significativa tra l'area mesangiale e la proteinuria al follow-up e tra l'area interstiziale e la cellularità interstiziale rispetto e la creatinina al follow-up (Figg. 10, 11). Lo studio comparativo delle biopsie renali prima e dopo trattamento ha mostrato un significativo incremento sia dell'area mesangiale sia dell'area interstiziale ed una significativa riduzione del rapporto lume/parete arteriolare nei pazienti del gruppo con mancata remissione.

Durante il trattamento non sono stati evidenziati effetti collaterali o indesiderati tali da richiedere la sospensione della terapia.

\section{Discussione}

Il sistema VMC consente un'analisi istomorfometrica oggettiva e confrontabile, che permette di ottenere interessanti correlazioni tra parametri clinicolaboratoristici ed istologici. Abbiamo infatti applicato il sistema in un nostro studio retrospettivo su 20 casi di glomerulonefrite sclerosante focale (17) ed i risultati hanno mostrato correlazione significativa tra l'indice di ischemia e di sclerosi e parametri laboratoristici, cioè l'indice di ischemia si correla con la proteinuria, VES e colesterolo totale, l'indice di sclerosi si correla con il numero delle piastrine. I nostri risultati, pur se ottenuti su una popolazione statisticamente non molto ampia, pongono interessanti interrogativi sulla genesi della glomerulonefrite sclerosante focale e sui fattori che possono essere indicatori di una prognosi più sfavorevole. Abbiamo applicato inoltre l'analisi istomorfometrica in un nostro studio retrospettivo su un gruppo di 27 soggetti anziani (età maggiore di 65 anni) confrontati con due gruppi di controllo rappresentati da 30 pazienti adulti (età 16-64 anni) e 25 pazienti in età pediatrica (età inferiore ai 16 anni) (18). Tutti i pazienti erano affetti da glomerulonefrite primitiva con sindrome nefrosica. L'analisi istomorfometrica ha mostrato simili lesioni glomerulari per ciascun tipo istologico di glomerulonefrite, sebbene le lesioni vascolari e tubulo-interstiziali fossero significativamente $(\mathrm{p}<0.05)$ più pronunciate nel gruppo dei pazienti anziani, specialmente nei casi di glomerulonefrite membrano-proliferativa.

L'analisi dei risultati del presente lavoro indica che l'associazione di una terapia cortisonica ed immunosoppressiva con ciclofosfamide è utile nel trattamento della glomerulonefrite membranosa idiopatica. La classificazione della glomerulonefrite membranosa in quattro stadi secondo Churg (19) non è in grado di far prevedere la risposta positiva al trattamento come dimostrato da una notevole percentuale di remissione completa in pazienti con stadio 4 e la possibilità di mancata risposta alla terapia anche in pazienti con lesioni modeste a carico della membrana basale glomerulare (stadio 1 e 2) (Fig. 9). Ciò è dovuto al fatto che nella classificazione di Churg non sono presi in considerazione il grado di interessamento tubulo-interstiziale e della compromissione mesangiale e vascolare che viceversa nel nostro lavoro sono emersi come elementi discriminanti tra il gruppo di responders e non responders al trattamento. Anche i parametri laboratoristici presi singolarmente non sono in grado di far prevedere l'evolutività della nefropatia, infatti non vi è una correlazione diretta tra proteinuria/die pre- e post-trattamento e creatininemia pre- e post-trattamento.

\section{Conclusioni}

Il sistema di videomicroscopia computerizzata da noi utilizzato si è rivelato utile nell'individuazione dei parametri istologici predittivi di evolutività e nella loro quantizzazione garantendo risultati riproducibili e confrontabili. Tale metodica può consentire di verifica- re, in biopsie seriate nel tempo, l'efficacia o meno di un determinato protocollo terapeutico attraverso la valutazione delle modificazioni dei parametri morfometrici.

L'uso di sistemi computerizzati nell'analisi istomorfometrica ha consentito peraltro un notevole risparmio nel tempo di esecuzione dell'esame rendendo possibile l'applicazione routinaria del1'istomorfometria nella diagnostica bioptica renale. 


\section{BIBLIOGRAFIA}

1. Ferrario F, Barbiano di Belgiojoso G, Cagnoli L et al. Le glomerulonefriti primitive e secondarie. Milano: Wichtig Editore, 1991.

2. Glassock JR, Adler SG, Ward $\mathrm{HJ}$ et al. Primary Glomerular Diseases. In: Brenner BM, Rector FC, eds. The Kidney. 4th ed. Philadelphia: WB Saunders Company 1991: 1182-279.

3. Ponticelli C, Banfi G, Tarantino A et al. Le glomerulopatie primitive. Syllabus di Nefrologia. Vol. 1. Milano: Wichting Editore, 1987.

4. Palmieri PF, Summa R, Di Liberato $\mathrm{L}$ et al. Nuove prospettive nel trattamento delle glomerulonefriti. GIN 1992; 9 S-2: 22.

5. Aparicio SR, Woolgar AE, Aparico SA et al. An ultrastructural morphometric study of membranous glomerulonephritis. Nephrol Dial Transplant. 1986; 1: 22-30.

6. Dische FE, Anderson VE, Keane SJ et al. Incidence of thin membrane nephrology: morphometric investigation of a population sample. J Clin Pathol 1990; 43: 457-60.

7. Fabbri PL, Campari A, Davoli $P$ et al. Applicazioni di elaboratori di immagine alla caratterizzazione di materiali e di dispositivi: alcune esperienze. Atti 85 Riunione Annuale Ass. Elettronica Ital. Riva del Garda 1984: 1-6.

8. Leonelli M, Furci L, Lusvarghi E. L'elaborazione elettronica di un'immagine nella diagnostica istopatologica renale: valutazione quantitativa di depositi evidenziati con immunoperossidasi. GIN 1988; 5: 107-12.

9. Collaborative study of the adult idiopathic nephrotic syndrome. A controlled study of short term prednisone treatment in adults with membranous nephropathy. N Engl J Med 1979; 301: 1301-6.
10. Cattran DC, Delmore T, Roscoe $\mathrm{J}$ et al. A randomized controlled trial of prednisone in patients with idiopathic membranous nephropathy. N Engl J Med 1989; 320; 210-5.

11. Cameron JS, Healy MJR, Adu D. The Medical Research Council trial of short term high dose alternate day prednisolone in idiopathic membranous nephropathy with a nephrotic syndrome in adults. Q $\mathbf{J}$ Med 1990; 74: 133-56.

12. Ponticelli C, Zucchelli P, Passerini $\mathrm{P}$ et al. A randomized trial of methylprednisolone and chlorambucil in idiopathic membranous nephropathy. N Engl J Med 1989; 320: 8-13.

13. Ponticelli C, Zucchelli P, Passerini $\mathrm{P}$, Cesani $\mathrm{B}$ and the Italian Idiopathic Membranous Nephropathy Treatment Study Group. Methyl prednisolone plus chlorambucil as compared with methyl prednisolone alone for the treatment of idiopathic membranous nephropathy. N Engl J Med 1992; 327: 599-603.

14. Mathieson PW, Turner AN, Maidment CGH, Evans DJ, Rees AJ. Prednisolone and chlorambucil treatment in idiopathic membranous nephropathy with deteriorating renal function. Lancet 1988; 2: 869-72.

15. West ML, Jindal KK, Bear RA, Goldstein MB. A controlled trial of cyclophosphamide in patients with membranous glomerulonephritis. Kidney Int 1987; 32: 579-84.

16. Palmieri PF, Santarelli $\mathrm{P}$, Greco V, Mastropierro G, Stuard S, Albertazzi A. Glomerulonefrite membranosa: follow-up a 30-60 mesi in 73 pazienti trattati con metilprednisolone e ciclofosfamide. XXXV Congresso Nazionale S.I.N., Bari 18-21 maggio 1994; 29.

17. Palmieri PF, Stuard S, Santarelli $\mathrm{P}$ et al. Evoluzione della glomerulonefrite sclerosante focale: fattori laboratoristici ed istologici di rischio. GIN 1992; 9 S-2: 22.
18. Palmieri PF, Bonomini M, Di Mizio G et al. Nephrotic syndrome in the elderly: a retrospective study in steroid treated patients. Contrib Nephrol Basel, Karger 1993; 105: 58.

19. Churg J, Sobin LH. Renal Disease. Classification and Atlas of Glomerular Diseases. Igaku Shoin, Tokio 1982; 54-65. 